\title{
Involvement of the human cerebellum during habituation of the acoustic startle response: A PET study
}

\begin{abstract}
Dagmar Timmann, Christina Musso, Florian P Kolb, Michel Rijntjes, Markus Jüptner, Stefan P Müller, Hans Christoph Diener, Cornelius Weiller
\end{abstract}

\begin{abstract}
The present study investigated the involvement of the human cerebellum in the habituation of the acoustic startle response using PET. The startle response was elicited in seven young, healthy subjects by a tone presented via headphones. Startle responses were recorded from the right sternocleidomastoid muscle. Regional cerebral blood flow (rCBF) was assessed in nine scans and one startle stimulus was applied during each scan. The reduction of size of the sternocleidomastoid muscle response was correlated with changes in $\mathrm{rCBF}$ during the ongoing process of startle response habituation. A significant decrease of $\mathrm{rCBF}$ was found in the medial cerebellum. These data are consistent with an involvement of the medial parts of the human cerebellum in non-associative learning as proposed by previous animal studies.

(F Neurol Neurosurg Psychiatry 1998;65:771-773)
\end{abstract}

Keywords: cerebellum; habituation; startle response; positron emission tomography

University of Munich, Pettenkoferstraße 12, 80336 Munich, Germany

F P Kolb

Department of Neurology,

Friedrich-Schiller-

University of Jena,

Philosophenweg 3 07740 Jena, Germany

C Musso

$M$ Rijntjes

C Weiller

Correspondence to: Dr Dagmar Timmann, Department of Neurology, University of Essen, Hufelandstraße 55, 45122 Essen, Germany. Telephone 0049201723 2460; fax 009 201723 5901; email

Dagmar.Timmann@

uni-essen.de

Received 15 December 1997 and in revised form

20 April 1998

Accepted 23 April 1998

within the lower brain stem. ${ }^{56}$ Animal studie have shown that the medial cerebellum is part of the essential circuitry for long term habituation of the acoustic startle response. ${ }^{6-8}$ So far, the role of the cerebellum in habituation of the startle response has not been investigated in humans. In the present study, involvement of the human cerebellum in habituation of the acoustic startle response was investigated in healthy subjects using PET.

\section{Materials and methods}

With the approval of the local ethics committee the study was performed in seven healthy subjects (five men and two women; age from 25 to 63 years, mean 35.5 years) without any hearing loss, without neurological and orthopaedic diseases, and not receiving any medication. All subjects were right handed. All gave their informed consent.

\section{STARTLE RESPONSE HABITUATION}

Subjects were lying supine and with their eyes closed. Head position was maintained by use of an individually molded foam headrest to minimise involuntary head movements during the scans. The startle stimulus consisted of an auditory tone pulse $(1000 \mathrm{~Hz} ; 95 \mathrm{~dB} ; 50 \mathrm{~ms})$ applied bilaterally via headphones. This tone was superimposed on continuous white noise (50 dB SPL) to mask environmental noise.

Electromyography recordings were performed from the right sternocleidomastoid muscle with surface electrodes (sampling rate $=1 \mathrm{kHz}$ ). The EMG traces were amplified, full wave rectified and bandpass filtered (10 $\mathrm{Hz}<\mathrm{f}<10 \mathrm{kHz}$ ). Latency and integrated EMG activity (iEMG) were calculated after visual identification of the onset of the sternocleidomastoid muscle activity using a commercial analysis program (axograph $\left.{ }^{\circledR}\right)$. Due to difficulties in defining the end point of the sternocleidomastoid muscle burst the EMG integral was calculated using a fixed interval of $100 \mathrm{~ms}$ after the onset of the EMG burst. Sternocleidomastoid muscle iEMG was normalised by assigning an arbitrary value of $100 \%$ to each subject's iEMG in the first trial and referencing changes in their iEMG to that value. The ongoing process of habituation was defined as the trial by trial reduction in size of the sternocleidomastoid muscle response.
SCANNING METHODS

Regional cerebral blood flow (rCBF) was measured during nine consecutive scans using the $\mathrm{H}_{2}{ }^{15} \mathrm{O}$ injection technique with an ECAT 


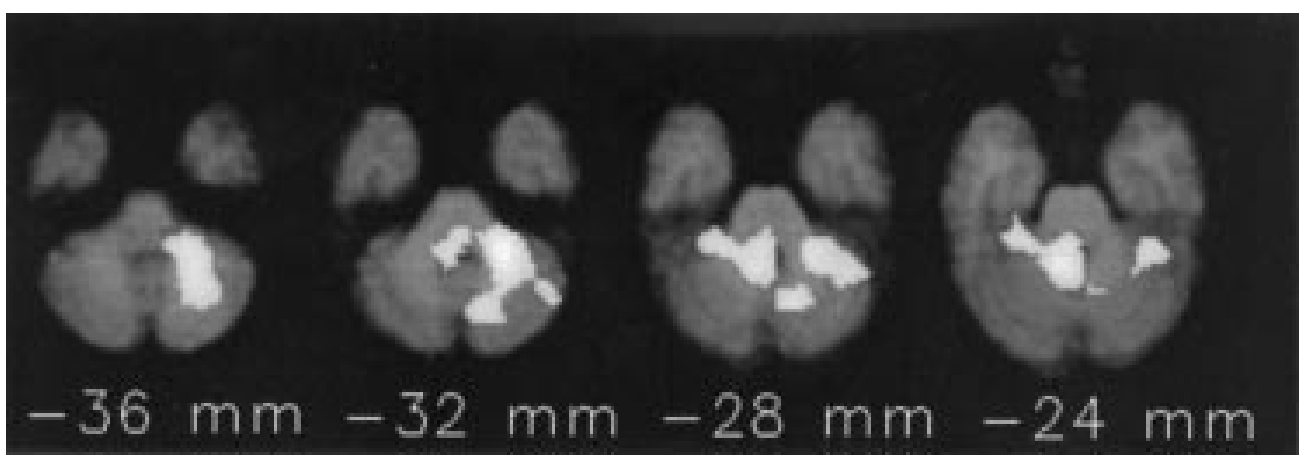

$t$ Maps showing all cerebellar areas correlating with the process of acoustic startle reflex habituation $(p<0.05)$. The average PET data $(n=7)$ were superimposed on to the MRI template. The highest correlation was seen in the intermediate parts of the left cerebellar hemisphere $(p=0.014)$.

953-15 PET scanner (CTI Inc, Knoxville, TN, USA) over 90 seconds. Ten seconds after the beginning of each scan one startle stimulus was applied (= total of nine auditory tone bursts). Data acquisition and analysis have been described previously in detail. ${ }^{4}$ In brief, assessment of significant $\mathrm{rCBF}$ change was performed using statistical parametric mapping (SPM, Wellcome Department of Cognitive Neurology, Queen Square, London, UK). The scans were transformed into the standard stereotactic anatomical space of Talairach and Tournoux. ${ }^{9}$ Because of the restricted field of view of our camera $(5.4 \mathrm{~cm})$ we decided to scan the lower parts of the brain which included the cerebellum. Scans were performed from -56 $\mathrm{mm}$ below the AC-PC line for cerebellar planes to $+24 \mathrm{~mm}$ above the AC-PC line for the frontal lobes, as the camera was tilted.

The rCBF of each test scan (1-9) was correlated pixel by pixel with normalised sternocleidomastoid muscle iEMG of each individual subject elicited by the startle stimulus during the corresponding scan using the general linear model. ${ }^{10}$ Significant changes in rCBF were assumed at $\mathrm{p}<0.05$ (corrected for multiple comparisons). Results are displayed as statistical parametric maps showing the significance level of areas of significant change of rCBF.

\section{Results}

Subjects habituated the acoustic startle response over the course of nine startle stimuli. Mean sternocleidomastoid muscle activity was reduced to $50.1 \%(\mathrm{SD}=19.0)$ of its initial size by the end of the PET session. Decrease of sternocleidomastoid muscle iEMG was roughly linear (linear regression: $R=0.70$, slope $=-3.5, \mathrm{p}<0.05)$.

The correlation analysis showed an area of significantly decreased blood flow in the cerebellum correlating with the ongoing pro-

Local maxima in the area of significant decrease $(p<0.05)$ of regional cerebellar blood flow correlating with startle response habituation

\begin{tabular}{lrlll}
\hline Cerebellar location & $x$ & $y$ & $z$ & $Z$ value \\
\hline Intermediate cerebellum (left) & -20 & -44 & -32 & $4.44^{\star}$ \\
Vermis (right) & 8 & -54 & -24 & 3.44 \\
Vermis (left) & -10 & -70 & -28 & 3.37 \\
Intermediate cerebellum (left) & -20 & -60 & -36 & 3.17 \\
Intermediate cerebellum (right) & 24 & -42 & -26 & 3.03 \\
Intermediate cerebellum (left) & -36 & -46 & -22 & 2.71 \\
Intermediate cerebellum (left) & -40 & -60 & -28 & 2.52 \\
\hline
\end{tabular}

${ }^{\star} \mathrm{p}=0.014$ cess of startle response habituation $(\mathrm{p}<0.05$, corrected for multiple comparisons). The cerebellar area of significantly decreasing $\mathrm{rCBF}$ extended from the vermis to intermediate regions of the cerebellar hemispheres predominately on the left side (figure). The highest correlation was seen in the left intermediate part of the cerebellum (maximum: $\mathrm{x}=-20 \mathrm{~mm}, \mathrm{y}=-44$ $\mathrm{mm}, \mathrm{z}=-32 \mathrm{~mm} ; \mathrm{Z}=4.44 ; \mathrm{p}=0.014$ (corrected for multiple comparisons)). Local maxima in the area of significant change of regional cerebellar blood flow correlating with startle response habituation are given in the table. There were no brain areas outside the cerebellum which showed significant change of $\mathrm{rCBF}$ correlating with the process of habituation. However, it has to be emphasised that the view of our camera was restricted $(5.4 \mathrm{~cm})$ and only the lower parts of the brain were scanned.

\section{Discussion}

We showed significant decreases in $\mathrm{rCBF}$ correlating with the process of habituation of the human acoustic startle response in the medial parts of the cerebellum. These data are consistent with an involvement of the human cerebellum in the habituation of the startle response.

The cerebellar vermis has been shown to be a key region of a circuit essential for the acquisition of long term habituation of the acoustic startle response in rats. ${ }^{6-8}$ However, medial cerebellar damage did not disrupt the basic neural circuitry for the acoustic startle response itself or the short term habituation mechanism which is presumed to be intrinsic to that pathway within the caudal brainstem. ${ }^{11}$ Because the cerebellum is not involved in the basic neural circuit for the acoustic startle response, involvement in the modulation of the response size seems likely. ${ }^{12}$ The significant decrease of cerebellar activity during the process of habituation might reflect a decrease in tonic activity on reticular neurons subserving startle responses during the process of habituation. ${ }^{13}$ In the present study, effects of short term and long term habituation were not separated with the subjects receiving a total of nine startle stimuli within one 2 hour test session. The significant change of $\mathrm{rCBF}$ might reflect activity related to short term or long term habituation.

It has to be emphasised that PET studies alone cannot tell us whether a particular locus 
is essentially involved in a function or informed about a function located elsewhere. The presented data of activity changes may most simply reflect the monitoring of peripheral events, one of the cerebellar tasks generally. ${ }^{14}$ As the size of muscular responses is diminished during the process of habituation the reduced activity in the cerebellum might simply reflect the reduced motor output.

The present study provides further evidence for the idea of functional compartments within the human cerebellum. A previous study from this laboratory showed a significant change of activity of the ipsilateral intermediate part of the human cerebellum during classical conditioning of the human flexion reflex. ${ }^{4}$ During startle response habituation, the area of significantly decreased $\mathrm{rCBF}$ included the vermis and adjacent intermediate parts of the cerebellum. The vermis seems predominantly to be involved in non-associative learning (habituation of the startle response) whereas different parts of the intermediate cerebellum contribute to non-associative and associative learning (classical conditioning). These findings are supported by animal studies which showed different localisation for specific aversive classical conditioning and non-specific fear habituation or conditioning: Cerebellar vermal lesions prevented long term startle reflex habituation and heart rate conditioning but not eyelid conditioning, whereas lateral cerebellar lesions prevented eyelid conditioning but not heart rate conditioning. ${ }^{16-815}$

In summary, involvement of the medial cerebellum in the habituation of the startle response has been demonstrated in human subjects by changes in $\mathrm{rCBF}$, monitored via PET. These data are consistent with an involvement of the human cerebellum in non-associative learning as suggested by previous animal studies. Further studies are needed to clarify if the human cerebellum is involved in short term, or long term habituation, or both, and whether its involvement is essential or not.

This paper was supported in part by a DFG-grant (Dagmar Timmann, Ti 239/2-1), by an EU grant (CT94/1261), and a grant from the Kuratorium ZNS Bonn. We gratefully thank H-G Elles for technical assistance, B Terschüren for her help in conducting the experiments, $\mathrm{S}$ Kiebel for his help in analysing the data, and $M$ Wei $\beta$ for preparing the manuscript.

1 Lavond DG, Kim JJ, Thompson RF. Mammalian brain substrates of aversive classical conditioning. Annual Review of Psychology 1993;44:317-42.

2 Bloedel JR, Bracha V. On the cerebellum, cutaneomuscular reflexess, movement control and the elusive engrams of memory [review]. Behav Brain Res 1995;68:1-44.

3 Woodruf-Pak D. Classical conditioning. International Review of Neurobiology 1997;41:341-66

4 Timmann D, Kolb FP, Baier C, et al. Cerebellar activation Timmann D, Kolb FP, Baier C, et al. Cerebellar activation
during classical conditioning of the human flexion reflex: a during classical conditioning of the hum

5 Brown P, Rothwell JC, Thompson PD, et al. New observations on the normal auditory startle reflex in man. observations on the normal
Brain 1991;114:1891-902.

6 Leaton RN, Supple WF. Cerebellar vermis: essential for long-term habituation of the acoustic startle response. Science 1986;232:513-5.

7 Leaton RN, Supple WF. Medial cerebellum and long-term habituation of acoustic startle in rats. Behav Neurosci 1991; 105:804-16.

8 Lopiano L, De'Sperati C, Montarolo PG. Long-term habituation of the acoustic startle response: role of the cerebellar vermis. f Neurosci 1990;35:79-84

9 Talairach J, Tournoux P. Co-planar stereotaxic atlas of the human brain. New York: Thieme, 1988.

10 Friston KJ, Holmes AP, Worsley KJ, et al. Statistical parametric maps in functional imaging: a general approach. parametric maps in functional imaging: a

11 Leaton RN, Cassella JV, Borszcz GS. Short term and long term habituation of acoustic startle response in chronic decerebrate rats. Behav Neurosci 1985;99:901-12

12 MacKay WA, Murphy JT. Cerebellar modulation of reflex gain. Prog Neurobiol 1979;13:361-417.

13 Eccles JC, Nicoll RA, Rantucci T, et al. Topographic studies on medial reticular nucleus. F Neurophysiol 1976;39:10918.

14 Kolb FP, Rubia FJ, Bauswein E. Comparative analysis of cerebellar unit discharge patterns in the decerebrate cat during passive movements. Exp Brain Res 1987;68:219-33.

15 Supple WF, Leaton RN. Lesions of the cerebellar vermis and cerebellar hemispheres: effect on heart rate conditioning in rats. Behav Neurosci 1990:104:934-47. 